\title{
Review \\ Hypoxia and oxidative stress in breast cancer Tumour hypoxia - therapeutic considerations
}

\author{
Kaye J Williams, Rachel L Cowen and lan J Stratford
}

Department of Pharmacy and Pharmaceutical Sciences, University of Manchester, Manchester, UK

Correspondence: Prof lan Stratford, Department of Pharmacy and Pharmaceutical Sciences, University of Manchester, Coupland III Building, Oxford Road, Manchester M13 9PT, UK. Tel: +44 161275 2487; fax +44 161275 2396; e-mail: ian.stratford@man.ac.uk

Received: 6 July 2001

Accepted: 18 July 2001

Published: 7 August 2001
Breast Cancer Res 2001, 3:328-331

(C) 2001 BioMed Central Ltd

(Print ISSN 1465-5411; Online ISSN 1465-542X)

\begin{abstract}
Conclusive research has shown that regions of acute/chronic hypoxia, which exist within the majority of solid tumours, have a profound influence on the therapeutic outcome of cancer chemotherapy and radiotherapy and are a strong prognostic factor of disease progression and survival. A strong argument therefore exists for assessing the hypoxic fraction of tumours, prior to patient treatment, and to tailor this treatment accordingly. Tumour hypoxia also provides a powerful physiological stimulus that can be exploited as a tumour-specific condition, allowing for the rationale design of hypoxia-activated anticancer drugs or novel hypoxia-regulated gene therapy strategies.
\end{abstract}

Keywords: bioreductive drugs, hypoxia, hypoxia responsive element, reductase enzymes

\section{Introduction}

Tissue hypoxia, owing to an inadequate blood supply, is a common feature of most solid tumours, and breast carcinoma is no exception. The accessibility of breast carcinomas has enabled the use of polarographic $\mathrm{O}_{2}$ needle electrodes to assess tumour oxygenation. Such studies have demonstrated an overall lower median $\mathrm{pO}_{2}$ level in malignant tumours compared with benign tumours and normal breast tissue. Electrode measurements obtained without the use of general anaesthesia have revealed median $\mathrm{pO}_{2}$ values of 23-28 mmHg for tumours, compared with $42 \mathrm{mmHg}$ for benign lesions and 54-65 mmHg for normal tissue [1,2]. Of all readings taken from breast cancers, 30-40\% fall below $10 \mathrm{mmHg}$, which is very rarely seen in normal tissue $[1,2]$. The study of Vaupel et al also revealed that nearly $40 \%$ of breast malignancies exhibit tumour regions with oxygen concentrations below that required for half-maximal radiosensitivity $\left(\mathrm{pO}_{2}<2.5 \mathrm{mmHg}\right)[1]$.

Radiotherapy and chemotherapy are both commonly incorporated into breast cancer treatment regimens.
Retrospective studies in other malignancies have determined that poor tumour oxygenation is the strongest prognostic indicator of radiotherapy treatment outcome [3-5]. In keeping with these findings, measurements of breast tumour $\mathrm{pO}_{2}$ distribution, prior to radiotherapy, have indicated that oxygen tension is an important modifier of radiation treatment outcome and is sufficient to predict local response [6]. In addition, the success of hypothermia as an adjuvant to radiation therapy for breast cancer treatment may be compromised in tumours with histopathology suggestive of a high level of chronic hypoxia [7]. The effects of hypoxia on the success of chemotherapy have not been clearly established. There is, however, direct evidence that hypoxic cells within tumour xenografts are refractile to chemotherapeutic agents and the quiescent nature of hypoxic cells may render them insensitive to agents that specifically target rapidly dividing cells $[8,9]$. Poor perfusion may limit drug dissemination to hypoxic regions. In addition, changes in gene expression to enable the survival of cells under hypoxic conditions may influence intracellular and extracellular $\mathrm{pH}$. A commonly

HIF-1 = hypoxia inducible factor $1 ; \mathrm{HRE}=$ hypoxia responsive element; TPZ = tirapazamine. 
prescribed chemotherapy treatment for breast cancer is cyclophosphamide, methotrexate, and 5-fluorouracil. Studies in murine tumours have shown increased response to cyclophosphamide when combined with agents that increase tumour oxygenation [10]. The uptake of 5 -fluorouracil by tumour cells grown in vitro can also be influenced by $\mathrm{pH}[11]$.

\section{Hypoxia as a therapeutic target}

Although hypoxia can be detrimental to treatment outcome, it can also be seen as a physiological abnormality that is restricted to the tumour and is therefore exploitable. This has lead to the development of bioreductive drugs that are preferentially cytotoxic towards hypoxic cells. The prototype bioreductive agent is the quinonealkylating agent, mitomycin $\mathrm{C}$, that has been widely used in combination chemotherapy regimens for breast cancer. Aside from quinones, there are two other classes of bioreductive drugs: nitro compounds and benzotriazine di- $N$ oxides. Of particular clinical interest in the latter group is tirapazamine, SR4233 (TPZ), which is in phase II/III clinical trials in combination with cisplatin. TPZ has shown increased treatment efficacy compared with cisplatin alone in malignant melanoma and non-small-cell lung cancer, and also may be of benefit when combined with both radiotherapy and cisplatin in head and neck cancer [12]. Although not extensively studied for the treatment of breast cancer, partial responses to combined TPZ/cisplatin treatment have been seen in phase I breast cancer patients [13].

The toxicity of quinones results from their one electron reduction to a semi-quinone free radical that covalently interacts with and damages DNA. In the presence of oxygen, this free radical can be oxidised back to the quinone concomitantly generating superoxide. Alternatively, two electron transfer may directly reduce quinones, or the semi-quinone can be further reduced, to generate a hydroquinone that can also be toxic. Nitro prodrugs are reduced by a series of single electron transfers with one, two, four and six electron reduction generating active species. Again the toxic nitroradical (one electron reduced) can be re-oxidised to the parent compound to release superoxide. Benzotriazine di- $N$-oxides are also reduced to a one electron reduced free radical. This free radical can be recycled by oxygen to generate superoxide; however, unlike the covalent interaction with DNA exhibited by the free radical species of quinones and nitro compounds, the $N$-oxide free radical is thought to act as an oxidising radical that abstracts hydrogen from the bases within DNA.

Although the precise activation pathway for these compounds differs, the compounds undergo reduction by endogenous enzymes to their cytotoxic moiety in aerobic and hypoxic cells but are only cytotoxic under hypoxia as a result of their back oxidation in the presence of oxygen (futile cycling). Many endogenous (one and two electron reducing) enzymes have been implicated in bioreductive drug activation: cytochrome P450s; cytochrome P450 reductase, xanthine oxidase and cytochrome $b_{5}$ reductase (one electron reducing enzymes); DT-diaphorase (two electron reducing enzyme); and carbonyl reductase (one and two electron reducing). These enzymes act to varying degrees and at different stages in a given prodrug activation.

Studies have shown that activity levels of carbonyl reductase and DT-diaphorase can be significantly elevated within human tumour biopsies, whereas cytochrome P450 reductase and cytochrome $b_{5}$ reductase levels were equivalent to those in normal tissue $[14,15]$. A more comprehensive analysis of the reductase status of tumours would allow for the rationale drug design of prodrugs tailored to the enzyme profile of the tumour. Alternatively, gene therapy can be exploited to achieve the specific overexpression of these enzymes within the hypoxic region of the tumour.

\section{Hypoxia-regulated gene therapy}

In the same way that hypoxia provides the physiological stimulus for bioreductive drug activation, it can also act as a trigger for specific gene transcription. Cell survival in response to hypoxic stress is dependent on the upregulation of genes supporting anaerobic metabolism and new blood vessel recruitment. This hypoxic gene activation is mediated through the transcription factor hypoxia inducible factor 1 (HIF-1). Recent studies have implicated a crucial role for HIF-1 in breast cancer progression. HIF-1 overexpression is commonly seen, with higher expression levels correlating with more aggressive disease [16]. Common to all HIF-1 responsive genes is a hypoxia responsive element (HRE). Introducing this HRE sequence into a gene delivery vehicle affords hypoxic regulation of the expressed gene. The potential of hypoxia selective gene therapy for cancer was first exemplified by the work of Dachs et al [17]. Using HRE-regulated expression of the suicide gene cytosine deaminase, Dachs et al demonstrated hypoxia-specific sensitisation of tumour cells to the prodrug 5-fluorocytosine. Subsequent studies have similarly demonstrated the efficacy of HRE-regulated herpes simplex virus thymidine kinase activity in ganciclovir sensitisation [18]. Although this approach offers a level of selectivity to standard gene-directed enzyme prodrug therapy, it can be further enhanced when used in combination with bioreductive drug treatment. Making hypoxia a prerequisite for both gene expression and drug activation may reduce cytotoxicity to healthy tissues (Fig. 1).

Reductase enzyme/prodrug pairings of bacterial nitroreductase/CB1954 [19], horseradish peroxidase/indole3-acetic acid [20], human cytochrome p450s/AQ4N 
Figure 1

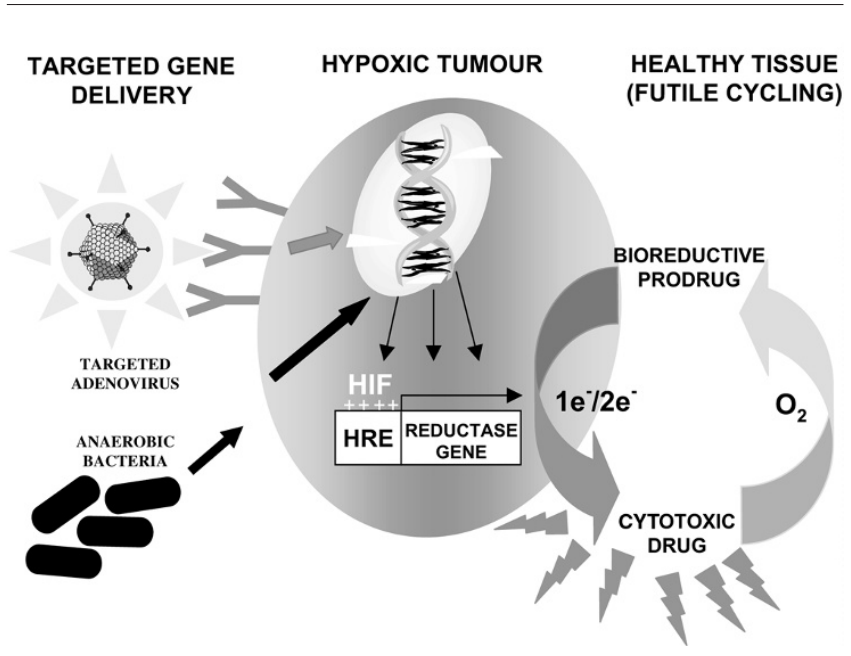

Hypoxia-regulated gene therapy. Hypoxia may be exquisitely exploited to achieve selective gene delivery, gene expression and bioreductive prodrug activation specifically within hypoxic tumour regions. Viral delivery vehicles can be specifically targeted to surface receptors upregulated on hypoxic cells or obligate anaerobes can be harnessed as vectors for their innate ability to colonise these regions. Making hypoxia a prerequisite for both gene expression and drug activation may reduce cytotoxicity to healthy tissues that can be achieved by combining hypoxia responsive element (HRE)-driven gene expression of a reductase gene with a bioreductive drug. This will allow the specific activation of the bioreductive drug to a free radical DNA damaging species within hypoxic cells that is free to diffuse to other hypoxic cells to exert a bystander effect. Diffusion to healthy aerobic tissues, in contrast, will result in re-oxidation of the drug to its non-toxic prodrug. HIF, hypoxia inducible factor $1 ; \mathrm{e}^{-}$, electron.

[21], DT-diaphorase/quinone EO9 [22] and human NADPH:cytochrome c p450 reductase/TPZ $[23,24]$ have been proposed. Gene delivery of cytochrome p450 reductase into breast tumour cell lines increases the efficacy of TPZ treatment in vitro [24]. Glioma tumours constitutively expressing both cytochrome p450 2B6 and cytochrome p450 reductase showed enhanced tumour growth delay when treated with cyclophosphamide (metabolised by cytochrome p450 2B6) and TPZ; however, systemic toxicity was also seen [25]. This may be potentially overcome by the use of a HRE to restrict expression to the hypoxic tumour environment. A particularly attractive refinement to this strategy for the treatment of breast cancer is the use of dual specificity promoters combining hypoxic regulation with tissue-specific regulation. The oestrogen receptor status of breast tumours identifies a cohort of tumours that have the potential to mount a transcriptional response to oestrogen. This is mediated through oestrogen responsive elements that have been successfully combined with HRE elements to give oestrogen and hypoxic transcriptional control of a pro-apoptotic gene [26]. Other breast-specific promoters, for example erbB-2 [27], could be used in conjunction with a HRE in this approach.
The limited clinical success of cancer gene therapy to date has been attributed to a lack of tumour-specific transduction. Clinically used viral vectors such as adenovirus have also been associated with preferential uptake by the liver and inherent immunogenicity. This necessitates the development of better, targeted delivery vehicles that could ideally be given systemically. This has lead to the ingenious application of obligate anaerobic bacteria as vectors, which selectively localise and proliferate within hypoxic cells when administered systemically. Non-pathogenic bacteria of the genera bifidobacterium and clostridia have been used to this effect $[19,28]$. Clostridia have been successfully used, allowing systemic delivery of several suicide genes including nitroreductase. An alternative approach has been to target existing viral vectors to hypoxic tumour cells. Viral vectors enter cells by receptormediated endocytosis, which can be specifically directed to receptors that exhibit elevated levels within hypoxic regions of a tumour. Combined with the use of polymer coating, which has the advantage of reducing the innate immunogenicity, vascular endothelial growth factor peptide coated adenovirus has been re-directed to the vascular endothelial growth factor receptor [29]. Macrophages that are known to migrate to hypoxic regions of tumours have also been exploited as a potential delivery vehicle of HRE-driven therapeutic genes [30].

\section{Conclusions}

Tumour hypoxia provides a unique opportunity for selective exploitation to achieve success in cancer therapy. It can be harnessed as a trigger for tumour selective drug activation and therapeutic gene expression, to be used as a powerful adjunct to conventional therapy where hypoxia plays an adverse role in treatment outcome. Although many of the gene therapy approaches are in their infancy, the potential advantage that may be gained through the use of a hypoxia-mediated therapeutic provides a strong rationale for assessing the oxygen status of breast tumours prior to treatment. The polarographic needle electrode approach is presently seen as the 'gold standard' for the measurement of tumour oxygenation. More readily available alternative methods for ascertaining hypoxic fraction are currently being developed. Use of the bioreductive marker pimonidazole is currently being evaluated in cancer patients. Pimonidazole is preferentially metabolised by hypoxic cells, and the pimonidazole adducts formed can be detected in sections or disaggregated tumour samples using antibodies. Pimonidazole binding has shown excellent concordance with established methodologies for analysing hypoxia in murine tumours [31]. The expression of numerous endogenous hypoxia-regulated proteins, including carbonic anhydrase IX and glucose transporter1 , is being evaluated for their potential use as surrogate markers for hypoxia in the clinical setting [32]. Studies have already demonstrated that carbonic anhydrase IX upregulation in breast cancer is associated with poor 
tumour outcome [33]. With the advent of non-invasive approaches to analyse tumour hypoxia [34], utilising standard imaging techniques already in the clinic, the specific tailoring of therapy regimes dependent on tumour oxygen status will provide unprecedented selectivity and therapeutic advantage for cancer treatment.

\section{References}

1. Vaupel P, Schlenger K, Knoop C, Hockel M: Oxygenation of human tumors: evaluation of tissue oxygen distribution in breast cancers by computerized $\mathrm{O}_{2}$ tension measurements. Cancer Res 1991, 51:3316-3322.

2. Hohenberger P, Felgner C, Haensch W, Schlag PM: Tumor oxygenation correlates with molecular growth determinants in breast cancer. Breast Cancer Res Treat 1998, 48:97-106.

3. Gatenby RA, Kessler HB, Rosenblum JS, Coia LR, Moldofsky PJ, Hartz WH, Broder GJ: Oxygen distribution in squamous cell carcinoma metastases and its relationship to outcome of radiation therapy. Int J Radiat Oncol Biol Phys 1988, 14:831-838.

4. Hockel M, Schlenger K, Aral B, Mitze M, Schaffer U, Vaupel P: Association between tumor hypoxia and malignant progression in advanced cancer of the uterine cervix. Cancer Res 1996, 56:4509-4515.

5. Brizel DM, Sibley GS, Prosnitz LR, Scher RL, Dewhirst MW: Tumor hypoxia adversely affects the prognosis of carcinoma of the head and neck. Int J Radiat Oncol Biol Phys 1997, 38: 285-289.

6. Okunieff P, Hoeckel M, Dunphy EP, Schlenger K, Knoop C, Vaupel $P$ : Oxygen tension distributions are sufficient to explain the local response of human breast tumors treated with radiation alone. Int J Radiat Oncol Biol Phys 1993, 26:631-636.

7. Lyng $H$, Rofstad EK: Treatment failure following sequential thermo-radiotherapy of locally advanced breast carcinoma occurs primarily in poorly vascularized tumors. Oncology 1995, 52:443-447.

8. Hill RP, Stanley JA: The response of hypoxic B16 melanoma cells to in vivo treatment with chemotherapeutic agents. Cancer Res 1975, 35:1147-1153.

9. Teicher BA, Lazo JS, Sartorelli AC: Classification of antineoplastic agents by their selective toxicities toward oxygenated and hypoxic tumor cells. Cancer Res 1981, 41:73-81.

10. Teicher BA: Hypoxia and drug resistance. Cancer Metastasis $\operatorname{Rev} 1994,13: 39-68$

11. Ojugo AS, McSheehy PM, Stubbs M, Alder G, Bashford CL, Maxwell RJ, Leach MO, Judson IR, Griffiths JR: Influence of pH on the uptake of 5-fluorouracil into isolated tumour cells. $\mathrm{Br} J$ Cancer 1998, 77:873-879.

12. Denny WA, Wilson WR: Tirapazamine: a bioreductive anticancer drug that exploits tumour hypoxia. Expert Opin Investig Drugs 2000, 9:2889-2901.

13. Johnson CA, Kilpatrick D, von Roemeling R, Langer C, Graham MA, Greenslade D, Kennedy G, Keenan E, O'Dwyer PJ: Phase I trial of tirapazamine in combination with cisplatin in a single dose every 3 weeks in patients with solid tumors. J Clin Oncol 1997, 15:773-780

14. Marin A, Lopez de Cerain A, Hamilton E, Lewis AD, MartinezPenuela JM, Idoate MA, Bello J: DT-diaphorase and cytochrome $\mathrm{B} 5$ reductase in human lung and breast tumours. $\mathrm{Br} J \mathrm{Cancer}$ 1997, 76:923-929.

15. Lopez de Cerain A, Marin A, Idoate MA, Tunon MT, Bello J: Carbonyl reductase and NADPH cytochrome P450 reductase activities in human tumoral versus normal tissues. Eur $J$ Cancer 1999, 35:320-324.

16. Bos $\mathrm{R}$, Zhong $\mathrm{H}$, Hanrahan CF, Mommers EC, Semenza GL, Pinedo HM, Abeloff MD, Simons JW, van Diest PJ, van der Wall E: Levels of hypoxia-inducible factor-1 alpha during breast carcinogenesis. J Natl Cancer Inst 2001, 93:309-314.

17. Dachs GU, Patterson AV, Firth JD, Ratcliffe PJ, Townsend KMS, Stratford IJ, Harris AL: Targeting gene expression to hypoxic tumour cells. Nat Med 1997, 3:515-520.

18. Koshikawa N, Takenaga K, Tagawa M, Sakiyama S: Therapeutic efficacy of the suicide gene driven by the promoter of vascular endothelial growth factor gene against hypoxic tumour cells. Cancer Res 2000, 60:2936-2941.
19. Lemmon MJ, van Zijl P, Fox ME, Mauchline ML, Giaccia AJ, Minton NP, Brown JM: Anaerobic bacteria as a gene delivery system that is controlled by the tumor microenvironment. Gene Ther 1997, 4:791-796

20. Greco O, Folkes LK, Wardman P, Tozer GM, Dachs GU: Development of a novel enzyme/prodrug combination for gene therapy of cancer: horseradish peroxidase/indole-3-acetic acid. Cancer Gene Ther 2000, 7:1414-1420.

21. Patterson LH, McKeown SR, Robson T, Gallagher R, Raleigh SM, Orr S: Antitumour prodrug development using cytochrome P450 (CYP) mediated activation. Anticancer Drug Des 1999, 14:473-486.

22. Warrington KH Jr, Teschendorf C, Cao L, Muzyczka N, Siemann DW: Developing VDEPT for DT-diaphorase (NOO1) using an AAV vector plasmid. Int J Radiat Oncol Biol Phys 1998, 42:909912.

23. Patterson AV, Barham HM, Chinje EC, Adams GE, Harris AL, Stratford IJ: Importance of $\mathbf{P} 450$ reductase activity in determining sensitivity of breast tumour cells to the bioreductive drug, tirapazamine (SR 4233). Br J Cancer 1995, 72:1144-1150.

24. Patterson AV, Saunders M, Chinje EC, Talbot DC, Harris AL, Stratford IJ: Overexpression of human NADPH:cytochrome $c$ (P450) reductase confers enhanced sensitivity to both tirapazamine (SR4233) and RSU1069. Br J Cancer 1997, 76: 1338-1347.

25. Jounaidi Y, Waxman DJ: Combination of the bioreductive drug tirapazamine with the chemotherapeutic prodrug cyclophosphamide for P450/P450-reductase-based cancer gene therapy. Cancer Res 2000, 60:3761-3769.

26. Hernandez-Alcoceba R, Pihalja M, Nunez G, Clarke MF: Evaluation of a new dual-specificity promoter for selective induction of apoptosis in breast cancer cells. Cancer Gene Ther 2001, 8: 298-307.

27. Pandha HS, Martin LA, Rigg A, Hurst HC, Stamp GW, Sikora K, Lemoine NR: Genetic prodrug activation therapy for breast cancer: A phase I clinical trial of erbB-2-directed suicide gene expression. J Clin Oncol 1999, 17:2180-2189.

28. Yazawa K, Fujimori M, Amano J, Kano Y, Taniguchi S: Bifidobacterium longum as a delivery system for cancer gene therapy: selective localization and growth in hypoxic tumors. Cancer Gene Ther 2000, 7:269-274.

29. Fisher KD, Stallwood Y, Green NK, Ulbrich K, Mautner V, Seymour LW: Polymer-coated adenovirus permits efficient retargeting and evades neutralising antibodies. Gene Ther 2001, 8:341-348.

30. Griffiths L, Binley K, lqball S, Kan O, Maxwell P, Ratcliffe P, Lewis C, Harris A, Kingsman S, Naylor S: The macrophage - a novel system to deliver gene therapy to pathological hypoxia. Gene Ther 2000, 7:255-262.

31. Raleigh JA, Chou SC, Arteel GE, Horsman MR: Comparisons among pimonidazole binding, oxygen electrode measurements, and radiation response in $\mathrm{C} 3 \mathrm{H}$ mouse tumors. Radiat Res 1999, 151:580-589.

32. Airley R, Loncaster J, Davidson S, Bromley M, Roberts S, Patterson A, Hunter R, Stratford I, West C: Glucose transporter glut-1 expression correlates with tumor hypoxia and predicts metastasis-free survival in advanced carcinoma of the cervix. Clin Cancer Res 2001, 7:928-934.

33. Chia SK, Wykoff CC, Watson PH, Han C, Leek RD, Pastorek J, Gatter KC, Ratcliffe PJ, Harris AL: Prognostic significance of a novel hypoxia regulated marker - carbonic anhydrase IX in invasive breast carcinoma. J Clin Onco/ 2001, in press.

34. Yang DJ, Ilgan S, Higuchi T, Zareneyrizi F, Oh CS, Liu CW, Kim EE, Podoloff DA: Noninvasive assessment of tumor hypoxia with 99mTc labeled metronidazole. Pharm Res 1999, 16:743750 . 